\title{
Asymmetric Anomalous Diffusion: an Efficient Way to Detect Memory in Time Series
}

\author{
Paolo Grigolini ${ }^{1,2,3}$, Luigi Palatella ${ }^{1}$, Giacomo Raffaelli ${ }^{1}$ \\ ${ }^{1}$ Dipartimento di Fisica dell'Università di Pisa and INFM \\ Piazza Torricelli 2, 56127 Pisa, Italy \\ ${ }^{2}$ Istituto di Biofisica del Consiglio Nazionale delle Ricerche, \\ Area della Ricerca di Pisa, Via Alfieri 1, San Cataldo, \\ 56010, Ghezzano-Pisa, Italy \\ ${ }^{3}$ Center for Nonlinear Science, University of North Texas, P.O. Box \\ 311427, Denton, Texas 76203-1427
}

\begin{abstract}
We study time series concerning rare events. The occurrence of a rare event is depicted as a jump of constant intensity always occurring in the same direction, thereby generating an asymmetric diffusion process. We consider the case where the waiting time distribution is an inverse power law with index $\mu$. We focus our attention on $\mu<3$, and we evaluate the scaling $\delta$ of the resulting diffusion process. We prove that $\delta$ gets its maximum value, $\delta=1$, corresponding to the ballistic motion, at $\mu=2$. We study the resulting diffusion process by means of joint use of the continuous time random walk and of the generalized central limit theorem, as well as adopting a numerical treatment. We show that rendering asymmetric the diffusion process yields the significant benefit of enhancing the value of the scaling parameter $\delta$. Furthermore, this scaling parameter becomes sensitive to the power index $\mu$ in the whole region $1<\mu<3$. Finally, we show our method in action on real data concerning human heartbeat sequences.
\end{abstract}

\section{Introduction}

One of the basic expectations of the statistical analysis of time series of biological, sociological and financial interest [1] is that the process under study 
might depart from fully random behavior. Following the prescriptions of the authors of Refs. 22, 3] we use the time series to create a sort of random walk trajectory whose statistical properties are then carefully studied to detect the deviations from ordinary Brownian motion. To do so a given site of the time series is interpreted as the "time" at which the walker makes a jump. The intensity of the jump is determined by the value of the time series at that given site, and the walker jumps either forward or backward according to whether the sign of the value is positive or negative. The most natural way to establish a comparison between this walker and the ordinary random walker of statistical mechanics would be to compare the two resulting diffusion processes to one another, but this would imply that infinitely identical walkers are available. Unfortunately a time series means only one trajectory, thereby generating the problem of how to create a number of "independent" trajectories large enough to produce a diffusion process. The authors of Refs. [2, 3] addressed this problem with an ingenious technique called Detrended Fluctuation Analysis (DFA) which allows us to bypass this difficulty. The whole pseudo-random trajectory is divided into non-overlapping intervals of length $l$, each of which can be imagined as being a trajectory running for a "time" l. Thus we have a collection of a relatively large number of "independent" trajectories that can be used to produce the wanted diffusion process. These trajectories might be affected by a local trend and this local trend might result in an artificial deviation from ordinary Brownian motion. For this reasons the authors of Refs. [2, 3] for any interval evaluate the local bias and then record only the deviation from the local trend. The square root of the resulting variance, denoted by them with the symbol $F(l)$, is expected to yield for very large l's

$$
F(l) \propto l^{\delta}
$$

where $\delta$ denotes the scaling parameter, a crucial property to assess the departure from ordinary Brownian motion. In fact, ordinary Brownian motion should yield $\delta_{B}=0.5$ and the deviation of the observed value of $\delta$ from $\delta_{B}=0.5$ is considered to be a measure of the deviation from a totally random behavior.

It has to be pointed out that scaling is a property implying that

$$
p(x, l)=\frac{1}{l^{\delta}} F\left(\frac{x}{l^{\delta}}\right)
$$

and that in some special cases, discussed in this paper, the scaling value detected by means of Eq.(1) does not afford a satisfactory account of the 
property of Eq.(2). Furthermore, as we shall see, the scaling $\delta$, even if it reflects correctly the property of Eq.(2), might not disclose an exhaustive information on the dynamic source of the deviation from ordinary Brownian motion.

Here we illustrate a technique of analysis, called Diffusion Entropy (DE) method, originally applied to detect memory effects in time series of sociological interest四. We shall show that this technique can, in part, solve the earlier mentioned problems. We use a sequence of the same kind as that adopted in earlier work to mimic the statistical properties of DNA sequences [5]. This sequence consists of strings of extended length, filled with only +'s or -'s. We consider as significant event only the change of sign. Thus, this kind of sequence is changed into a new sequence realized by assigning the vanishing value to the sites with a sign identical to that of the following one. Only the sites with a sign opposite to that of the next site are assigned a non vanishing value, which is always the same positive quantity, $W$. Thus we obtain a sequence of rare events. When one of these events occurs, the random walker makes a jump of constant intensity, always in the same direction. We prove that the resulting diffusion process makes much more ostensible the correlated nature of the original time series.

We also show that this procedure makes faster the detection of the proper scaling parameter $\delta$, namely, a scaling value which is a fair reflection of the property of Eq.(22). As earlier mentioned, the methods determining scaling through variances might yield scaling parameters significantly departing from the ideal scaling value. This is the scaling that an artificial time series, whose asymptotic behavior is dictated by compelling theoretical arguments, is expected to reach in the long-time limit.

The outline of the paper is as follows. In Section 2 we describe the main properties of the DE method, and with the help of an artificial sequence we show that the observation of a symmetric diffusion process results in a slow convergence towards the emergence of the expected scaling parameter. Section 0 illustrates a theoretical approach to the scaling of the asymmetric diffusion process using two distinct procedures, the Continuous Time Random Walk (CTRW) and the Generalized Central Limit Theorem (GCLT), yielding the same result. This section also shows, with the help of the DE method applied to some artificial sequences, that the detection of the correct scaling is faster than in the case of symmetric diffusion. Finally, Section 1 shows our detection technique at work on real data. The illustrative example of Section 1 is based on a time series resulting from the record of human heart 
beats. A balance on the results obtained in this paper is made in Section 5 .

\section{Diffusion Entropy}

The main idea of this approach to scaling is remarkably simple. Let us consider a sequence of $M$ numbers $\xi_{i}$, with $i=1, \ldots, M$. The purpose of the DE algorithm is to establish the possible existence of a scaling, either normal or anomalous, in the most efficient way as possible, without altering the data with any form of detrending. Let us select first of all an integer number $l$, fitting the condition $1 \leq l \leq M$. This integer number will be referred to by us as "time". For any given time $l$ we can find $M-l+1$ sub-sequences defined by

$$
\xi_{i}^{(s)} \equiv \xi_{i+s}, \quad s=0, \ldots, M-l .
$$

For any of these sub-sequences we build up a diffusion trajectory, labelled with the index $s$, defined by the position

$$
x^{(s)}(l)=\sum_{i=1}^{l} \xi_{i}^{(s)}=\sum_{i=1}^{l} \xi_{i+s} .
$$

Let us imagine this position as referring to a Brownian particle that at regular intervals of time has been jumping forward or backward according to the prescription of the corresponding sub-sequence of Eq.(33). This means that the particle before reaching the position that it holds at time $l$ has been making $l$ jumps. The jump made at the $i$-th step has the intensity $\left|\xi_{i}^{(s)}\right|$ and

is forward or backward according to whether the number $\xi_{i}^{(s)}$ is positive or negative.

We are now ready to evaluate the entropy of this diffusion process. To do that we have to partition the $x$-axis into cells of size $\epsilon(l)$. When this partition is made we have to label the cells. We count how many particles are found in the same cell at a given time $l$. We denote this number by $N_{i}(l)$. Then we use this number to determine the probability that a particle can be found in the $i$-th cell at time $l, p_{i}(l)$, by means of

$$
p_{i}(l) \equiv \frac{N_{i}(l)}{(M-l+1)} .
$$


At this stage the entropy of the diffusion process at the time $l$ is determined and reads

$$
S_{d}(l)=-\sum_{i} p_{i}(l) \ln \left[p_{i}(l)\right]
$$

The easiest way to proceed with the choice of the cell size, $\epsilon(l)$, is to assume it independent of $l$ and determined by a suitable fraction of the square root of the variance of the fluctuation $\xi(i)$.

Before proceeding with the illustration of how the DE method works, it is worth making a comment on the way we use to define the trajectories. The method we are adopting is based on the idea of a moving window of size $l$ that makes the $s-t h$ trajectory closely correlated to the next, the $(s+1)-t h$ trajectory. The two trajectories have $l-1$ values in common. The DFA, on the contrary, is based on non-overlapping windows, and consequently trajectories with different labels are totally independent the one from the other. A motivation for our choice is that we are having in mind a possible connection with the Kolmogorov Sinai (KS) entropy [6, []. The KS entropy of a symbolic sequence is evaluated by moving a window of size $l$ along the sequence. Any window position corresponds to a given combination of symbols, and, from the frequency of each combination, it is possible to derive the Shannon entropy $S(l)$. The KS entropy is given by the asymptotic limit $\lim _{l \rightarrow \infty} S(l) / l$. We believe that the same sequence, analyzed with the DE method, at the large values of $l$ where $S(l) / l$ approaches the KS value, must yield a well defined scaling $\delta$. To realize this correspondence we carry out the determination of the DE using the same criterion of overlapping windows as that behind the KS entropy.

Details on how to deal with the transition from the short-time regime, sensitive to the discrete nature of the process under study, to the long-time limit where both space an time can be perceived as continuous, are given in Ref.[8]. Here we make the simplifying assumption of considering so large times as to make the continuous assumption valid. In this case the trajectories, built up with the above illustrated procedure, correspond to the following equation of motion:

$$
\frac{d x}{d t}=\xi(t)
$$

where $\xi(t)$ denotes the value that the time series under study gets at the $l-t h$ site. This means that the function $\xi(l)$ is depicted as a function of $t$, thought of as a continuous time $t=l$. In this case the Shannon entropy 
reads

$$
S(t)=-\int_{-\infty}^{\infty} d x p(x, t) \ln [p(x, t)]
$$

We assume

$$
p(x, t)=\frac{1}{t^{\delta(t)}} F\left(\frac{x}{t^{\delta(t)}}\right) .
$$

This is a generalization of the ordinary scaling assumption of Eq.(2), which, in fact, can be recovered by assuming $\delta(t)$ to be time independent so that we can denote it as $\delta$. For the sake of simplicity we make the simplifying assumption that $F(y)$ maintains its form, namely, that the statistics of the process are unchanged. This is questionable in the case where the scaling is a function of time. However, making this assumption we obtain the following results. Let us plug Eq.(9) into Eq.(8). After a simple algebra, we get:

$$
S(\tau)=A+\delta(\tau) \tau
$$

where

$$
A \equiv-\int_{-\infty}^{\infty} d y F(y) \ln [F(y)]
$$

and

$$
\tau \equiv \ln (t)
$$

It is evident that this kind of technique to detect scaling does not imply any form of detrending, and this is one of the reasons why some attention should be devoted to it. An interesting way to check the efficiency of this technique is realized by the study of the artificial sequence of Ref. [9]. This sequence is built up in such a way as to realize long sequences of either +'s or -'s. The probability of finding a sequence of only +'s or only -'s of length $t$ is given by

$$
\psi(t)=(\mu-1) \frac{T^{\mu-1}}{(t+T)^{\mu}} .
$$

Here we focus our attention on the condition $\mu<3$ and we raise the reader's attention on the interval $[2,3]$. In fact, this kind of sequence is the same as that adopted in earlier work [10] for a dynamic derivation of Lévy diffusion, which shows up when the condition $2<\mu<3$ applies. It corresponds to a particle travelling with constant velocity throughout the whole time interval corresponding to either only +'s or only -'s, and changing direction with no rest, at the end of any string with the same symbols. 
We will refer to this model as Symmetric Velocity Model (SVM). We know from the theory of Ref. [10 that the scaling of the resulting diffusion process when $2<\mu<3$ is

$$
\delta=\frac{1}{\mu-1} .
$$

Note, however, that this diffusion process has a finite propagation front, with ballistic peaks showing up at both $x=t$ and $x=-t$. The intensity of these peaks is proportional to the correlation function

$$
\Phi_{\xi}(t)=\left(\frac{T}{T+t}\right)^{\mu-2} .
$$

As a consequence of this fact, the whole distribution does not have a single rescaling. In fact, the distribution enclosed between the two peaks rescales with $\delta$ of Eq.(14) while the peaks are associated to $\delta=1$. Furthermore, it is well known 10 that the scaling of the second moment is given by

$$
\delta=\frac{4-\mu}{2} .
$$

Thus, it is expected that the scaling detected by the DE method might not coincide with the prediction of Eq.(14) for the whole period of time corresponding to the presence of peaks of significant intensity. We think that the Lévy scaling of Eq.(14) will show up at long times, when the peak intensity is significantly reduced. This conjecture seems to be supported by the numerical results illustrated in Fig.11. We see in fact that the scaling predicted by Eq.(14) is reached after an extended transient, of the order of about 20,000 in the scale of Fig.11. This time interval is about 2000 larger than the value assigned to the parameter T, of Eq.(13), which is, in fact, in the case of Fig.1, $\mathrm{T}=10$.

In conclusion, this section proves that the DE method applied to the SVM yields, for the scaling parameter $\delta$, the correct value of Eq.(14), rather than the value that would be obtained measuring the variance of the diffusion process, Eq.(16). However, the time necessary to make this correct value emerge is very large. Furthermore, as proved by the theory of Ref.[11], the adoption of SVM would make the scaling parameter $\delta$ insensitive to $\mu$ in the whole interval $[1,2]$. This means that the adoption of the DE method would not allow us to distinguish a process with $\mu$ very close to 1 from one with $\mu$ very close to 2 . In Section 3 we shall see how to overcome these limitations. 


\section{Asymmetric Diffusion}

Let us consider again the kind of artificial sequence that we analyzed in Section 2. Let us change perspective. Let us explicity focus on the events corresponding to changing sign. For this reason we build up a new sequence where the sites of the laminar region are given the vanishing value. This means that the states of constant velocity are here perceived as states of rest. The random walker can make a jump only at the moment when the time series analyzed in Section II changes signs. If at the moment of changing sign the walker makes a jump, of the same intensity, forward or backward, according to whether the sign change is positive or negative, we shall refer to this process as Symmetric Jump Model (SJM). The scaling corresponding to the SJM has been studied by Shlesinger in the pioneer paper of Ref. [12], which yields the following prescription when the condition $1<\mu<2$ applies,

$$
\delta=\frac{1}{2}(\mu-1) .
$$

When $\mu>2$, the theory of Ref. [12] predicts

$$
\delta=1 / 2 .
$$

We see that the whole region $\mu>2$, including that with $\mu<3$, would be indistinguishable from an ordinary Brownian diffusion. For this reason, in addition to focusing our attention on the events corresponding to the sign change, we decide to disregard whether the change of sign is from - to + or vice-versa. This generates a kind of asymmetric diffusion process that we term Asymmetric Jump Model (AJM). This section is devoted to the discussion of the AJM by means of two distinct theoretical approaches, both resulting in the same prediction, an interesting form of asymmetric Levy diffusion. Then we generate two artificial sequences corresponding to $\mu>2$ and $\mu<2$, respectively, and we analyze them by means of the DE method.

\subsection{Continuous Time Random Walk}

The first theoretical approach rests on the Continuous Time Random Walk (CTRW) of Montroll and Weiss [13]. This means that the distance between two nearest neighbor events, $\tau_{i} \equiv t_{i+1}-t_{i}$, is considered to be a random

number, described by the probability density $\psi(\tau)$. Note that we are here 
using the notation $\tau$ rather than $t$ to make clear to the reader the difference between the absolute time $t$ and the time distance between two nearest neighbor events. As earlier said, we assume that the random walker always moves in the same direction. Moreover, we also set the condition that the steps have constant length. Thus, the probability that a step of length $x$ is taken is given by

$$
\Pi(x)=\delta(x-W) .
$$

Applying to this case the CTRW formalism of Montroll and Weiss [13], we write for $p(x, t)$, the probability of the walker being at position $x$ at time $t$,

$$
p(x, t)=F^{-1} L^{-1} \hat{p}(k, s) \equiv F^{-1} L^{-1}\left[\frac{1-\hat{\psi}}{s} \frac{1}{1-\lambda(k) \hat{\psi}(s)}\right],
$$

where $\lambda(k)$ and $\hat{\psi}(s)$ are the Fourier and Laplace transform of $\Pi(x)$ and $\psi(t)$, respectively. Note that

$$
\lambda(k)=\exp (i k W) .
$$

By Taylor series expansion of Eq.(20) we get:

$$
\hat{p}(k, s)=\frac{1-\hat{\psi}}{s} \sum_{n=0}^{\infty} \hat{\psi}^{n} e^{i n k W}
$$

By evaluating the inverse Fourier transform of Eq.(22) we arrive at:

$$
\hat{p}(x, s)=\frac{1-\hat{\psi}}{s} \sum_{n=0}^{\infty} \hat{\psi}^{n} \delta(x-n W) .
$$

This means that for large distances we can adopt the following expression

$$
p(x, s)=[\hat{\psi}(s)]^{\frac{x}{W}} \frac{1-\hat{\psi}(s)}{s} .
$$

We shed light on the meaning of Eq.(24) with the following remarks. Let us denote by $\psi_{x}(t)$ the probability of making $x$ jumps up to time $t$, with the last jump occurring at $t$. Let us denote by $\Phi_{n}(t)$ the probability of making $n$ jumps at earlier times, $t^{\prime}<t$. The two functions are connected by the relation

$$
\Phi_{n}(t)=\int_{0}^{t} \psi_{n}\left(t^{\prime}\right) \Psi\left(t^{\prime}-t\right) d t^{\prime}
$$


where

$$
\Psi(t) \equiv \int_{t}^{\infty} \psi\left(t^{\prime}\right) d t^{\prime}
$$

Using the properties $\hat{\psi}_{n}(s)=[\hat{\psi}(s)]^{n}$ and $\hat{\Psi}(s)=(1-\hat{\psi}(s)) / s$, and Laplace transforming Eq.(25), we get

$$
\hat{\Phi}_{n}(s)=\hat{\psi}_{n}(s) \hat{\Phi}(s)=[\hat{\psi}(s)]^{n} \frac{1-\hat{\psi}(s)}{s} .
$$

We see that the result of Eq.(24) can be recovered by identifying $n$ with $x / W$, as it is legitimate to do when the number of jumps done is so large as to perceive $n$ as a continuous number.

Let us focus now our attention on the case where the distribution of waiting times, $\psi(t)$, has the following asymptotic behavior

$$
\psi(t) \approx \frac{\text { const }}{t^{\mu}},
$$

with $\mu>1$ so as to insure the normalization condition. Let us focus our attention on the case where

$$
1<\mu<2 \text {. }
$$

This is the region where jumps of intensity $x$ occurring with equal probability in both the positive and the negative direction of the $x$-axis, i.e. the SJM, would result 11] in a diffusion process with the scaling $\delta$ of Eq.(17). We now show that, as a benefit of adopting the AJM rather than the SJM, we derive an enhanced scaling parameter. In fact, for very small values of $s$,

$$
\hat{\psi}(s) \approx 1-c s^{\gamma}
$$

where

$$
\gamma=\mu-1
$$

This is the same property as that used by Zumofen and Klafter 11. By plugging Eq.(30) into Eq.(24) and by inverting the Laplace transform with the nice method of ref. 114, we obtain

$$
p(x, t) \approx \frac{t}{x^{\frac{1}{\gamma}+1}} L\left(\frac{t}{x^{\frac{1}{\gamma}}}, \gamma,-\gamma\right),
$$


where $L(y, \gamma,-\gamma)$ denotes a fully asymmetric Lévy stable law of index $\gamma 15$. It is straightforward to express this important result in the form of Eq.(9) with the time independent scaling

$$
\delta=\mu-1
$$

By comparing Eq.(33) to Eq.(17) it becomes evident the scaling enhancement produced by making the diffusion process asymmetric. In fact, at $\mu=3 / 2$ a transition is made from subdiffusion to superdiffusion.

\subsection{Generalized Central Limit Theorem}

The result of Section 3.1 can be recovered from within the attractive perspective of the Generalized Central Limit Theorem (GCLT) [16] in a form derived from properly adopting the procedure of Feller [17] to our purposes. To make closer the connection with the formalism of Feller let us assume $W=1$. The function $p(x, t)$ in this case is nothing but the probability for the walker to make $x$ steps up to time $t$, or, equivalently, the probability of occurrence of $x$ events up to time $t$. It has to be stressed that according to the perspective expressed by Refs. 18, 19, in accordance with the arguments of Gaspard and Wang [20], this way of proceeding can also be used to measure the complexity or randomness of the process under study, in the special case where the time series under study is totally memory-less. In fact, in this specific case the occurrence of any sporadic event does not have any connection with the earlier or subsequent events. It has to be pointed out that the kind of time series that we are having in mind in this paper is characterized by events of the same intensity, or made so by ignoring the real intensity of each event. If there is no correlation between intensity and time occurrence of the event, the simplifying assumption that the events have the same intensity does not have any significant consequence on the scaling of the resulting diffusion.

If the simplifying assumption applies, that no form of memory exists, in addition to the inverse power law nature of the waiting time distribution $\psi(t)$, we can safely apply the Feller arguments. Feller [17] studied the case where the survival probability $\Psi(t)$ has the following time asymptotic expression

$$
\Psi(t) \approx \frac{1}{t^{\gamma}} .
$$

The Feller notation is related to our notation by $\gamma=\mu-1$. In the article of Ref.[17], Feller studies bot the case $0<\gamma<1$ and the case $1<\gamma<2$, 
which refers to our two regimes $1<\mu<2$ and $2<\gamma<3$, respectively. In the former case, Feller finds the important property

$$
\operatorname{Pr}\left(N_{t} \geq w t^{\gamma}\right)=G_{\gamma}\left(w^{-1 / \gamma}\right)
$$

where, according to the notation used by Feller [17] $\operatorname{Pr}\left(N_{t}>y\right)$ denotes the probability that the number of events occurring at times $t^{\prime} \leq t$ is greater than $y$. The function $G_{\gamma}(x)$ denotes the stable law whose characteristics function is given by

$$
\hat{G}_{\gamma}(z)=\exp \left\{-|z|^{\gamma}\left[\cos \left(\frac{\pi \gamma}{2}\right)-i \sin \left(\frac{\pi \gamma}{2}\right) \operatorname{sgn}(z)\right] \Gamma(1-\gamma)\right\} .
$$

The symbol $\Gamma$ denotes the Euler function. The meaning of the function $G_{\gamma}(w)$ is made transparent by the case where the walker makes jumps in the same direction, with the probability $\Pi(x) \propto 1 / x^{\gamma}$, at regular interval of times. In this specific case, which is the case of an asymmetric Levy flight, the position of the random walker at time $t, X_{t}$, fulfills the property

$$
\operatorname{Pr}\left(X_{t} \leq w t^{1 / \gamma}\right)=G_{\gamma}(w)
$$

The function on the right hand side of Eq. (37) is the same as that appearing in the right hand side of Eq. (35). However, we know that it has to do with an asymmetric Levy process. Consequently, it is a stable law that the theory of 15] makes it possible to write under the form

$$
G_{\gamma}(w)=\int_{0}^{w} L(y, \gamma,-\gamma) \mathrm{d} y,
$$

where $L(y, \gamma,-\gamma)$ is the function defined in Section 3.1. We note that Eq. (35) can also be written as

$$
\int_{w t^{\gamma}}^{\infty} \mathrm{d} N_{t} p\left(N_{t}\right)=G_{\gamma}\left(w^{\gamma}\right) .
$$

By differentiating Eq. (39) with respect to $w$ and setting $N_{t}=x$ (the position of the walker at time $t$, labeled as $x$, is equal to the number of steps taken up to time $t$, namely, $N_{t}$ ) we get

$$
p(x, t) \approx \frac{t}{x^{\frac{1}{\gamma}+1}} L\left(\frac{t}{x^{\frac{1}{\gamma}}}, \gamma,-\gamma\right),
$$


which agrees with the scaling predicted by the CTRW arguments used in section 3.1 since it leads to the scaling parameter $\delta=\mu-1$.

In the case where $1<\gamma<2$ we are led by the corresponding Feller prescription [17 to express the probability distribution in the reference translating with the mean velocity (the inverse of the mean waiting time). With an argument similar to the one earlier used we obtain

$$
p(x, t) \approx \frac{1}{t^{\frac{1}{\gamma}}} L\left(\frac{x}{t^{\frac{1}{\gamma}}}, \gamma,-\gamma\right),
$$

and it is now easy to prove that this yields, for $2<\mu<3$, the scaling prescription

$$
\delta=\frac{1}{\mu-1} .
$$

Results are summarized in Tab. 1.

\subsection{Numerical Calculations}

This section is devoted to illustrating the results of numerical calculations that confirm the benefits of the strategy suggested in this paper. First of all with Fig. 2 we show that the AJM makes it possible to reach much earlier the correct Lévy scaling of Eq.(14). The numerical work shows that the time scale at which the DE method yields the correct scaling is, in this case, equal to about $2,000(T=10)$.

We also prove that the resulting scaling value is a genuine reflection of the property of Eq.(2). To do so, we express the diffusion process in the

reference framework moving with the velocity $T W /(\mu-2)$ corresponding to the theoretical prediction of section 3.2. Then we consider a reference time $t_{n_{0}}$ and later times $t_{n}>t_{n_{0}}$. For any time $t_{n}$ we consider the squeezing parameter $R(n)=\left(t_{n} / t_{n_{0}}\right)^{\delta}$, with $\delta$ given by the scaling detected by the DE method. The distance scale is squeezed by the quantity $R(n)$ and the ordinate scale is enhanced by the quantity $1 / R(n)$. If the scaling detected by the $\mathrm{DE}$ is correct and if this procedure is applied to the distribution corresponding to the time $t_{n}$ this scaling procedure should make it identical to the distribution corresponding to the time $t_{n_{0}}$. Fig. 3 shows that this is really so, since all the distributions at times $t_{n}$ are made to virtually coincide with the distribution at time $t_{n_{0}}$.

We have explored also the case $\mu<2$. Figure 4 shows the result of the DE method in this case, and Fig.5 shows the result of scaling back to earlier 
times the distributions corresponding to later times, in this case. The result is not as accurate as that illustrated by Fig.3. However, we judge it to be satisfactory, especially if we take into account the fact that the region $\mu<2$ corresponds to the case of extremely large time correlations, with an infinite sojourn time.

\section{The Method in Action in the Case of Real Sequences}

As an example of how this technique can be applied to real data we considered time series concerning human heartbeats. These series were taken from Physionet [21] and are a record of time intervals between successive beats. To apply our method we adopted a coarse graining procedure: times between beats were first multiplied by 10, with only the integer part of the resulting number taken into account. This sequence of integer numbers was then transformed into a new one according to the following prescription: we wrote 0 if the number was equal to the preceeding one, 1 if it was different. We note that this would have resulted almost in a sequence of only 1's, without any 0 , had we not adopted the coarse graining procedure. From this sequence we generated an asymmetric diffusion process (AJM) which we then studied by means of DE method described in the previous sections to derive the scaling parameter $\delta$. This corresponds to having a walker taking a step in a fixed direction when the time interval between successive beats, as seen on a coarse grained scale, differs from the preceding one. We also analyzed the probability distribution $\psi(t)$ of waiting times between the 1 's, which shows an inverse power-law behavior with index $\mu>2$. We thus expect the correct scaling to be predicted by Eq.(42) rather than by Eq.(33). Results obtained from different databases are reported in table 5, whose third column represents the value of $\mu$ we derive from $\delta$. An example for a particular database (Ltdb15814) is shown in Fig.6 where we derive the scaling $\delta$ using DE, and in Fig.7, where we show how the inverse power law of index $\mu=1+1 / \delta$ indeed fits the data used to obtain $\psi(t)$. 


\section{Concluding Remarks}

This paper is a sequel to that of Ref. [4], where the method of DE was applied for the first time. However, the emphasis here is on the detection of the scaling more than on the property that the authors of Ref. [1 1 referred to as memory. On the basis of the results of the present paper, the memory of Ref. [4] seems to have to do with the transition from the short-time to the longtime regime. The main result here reached, however, has to do with proving the convenience of recording the times at which events occur, an event being a sign change, regardless of whether it implies an increase or a decrease of the intensity signal. If we record the time at which the event occurs, and we interpret it as a jump of the random walker always in the same direction, with a fixed intensity, then the diffusion process becomes asymmetric, with the big benefit of enhancing the value of the scaling parameter in the interval $1<\mu<2$, and of making it sensitive to the power law index $\mu$ in the whole interval $1<\mu<3$. The AJM has to be compared to the SJM, a model that the theoretical analysis of the 1974 pioneer work of Shlesinger [12 shows to be insensitive to $\mu$ in the range $2<\mu<3$. The value of the scaling parameter $\delta$, stemming from the AJM, as earlier repeatedly pointed out, is enhanced with respect to that of the $\mathrm{SJM}$, in the whole interval $2<\mu<3$. If we compare the AJM to the SVM of Ref.[1], we find that it provides information on $\mu$ even in the interval $1<\mu<2$, where the SVM would steadily result in the ballistic prescription $\delta=1$.

It is interesting to notice that the AJM proposed by this paper emphasizes the phase transition character of a transformation moving from $\mu>2$ to $\mu<2$. As pointed out in Ref. [19], this is a transition from a stationary to a non-stationary regime. In fact, Ref. [19] shows that $\mu<2$ implies the lack of an invariant distribution, thereby making any observation carried out for a finite time equivalent to monitoring an out of equilibrium process. We are convinced that the systematic application of the prescriptions of this paper to studying time series generated by complex dynamic processes, might lead to discover these non-stationary cases.

The application to the real sequences of physiological interest of Section 4 4 shows that the detection of the scaling parameter $\delta$ is much more accurate than the direct evaluation of $\mu$ from the experimental data, the only ambiguity being the fact that the value of $\delta$ is compatible with both $\mu(1)=1+1 / \delta$ and $\mu(2)=1+\delta$. So, the experimental error affecting the direct determination of delta must only be smaller than the difference $\mu(1)-\mu(2)$. Only 
when the value of $\mu$ is very close to the transition value $\mu=2$, the adoption of the DE method to detect $\mu$ becomes ambiguous, and additional criteria must be found.

A possible efficient criterion could be given by the joint use of the SVM and AJM method. In fact, as clearly illustrated by Table 1, if the SVM yields $\delta=1$, this means that $\mu<2$. If, on the contrary, the SVM, within the statistical accuracy of the numerical procedure, yields the same scaling as the SVM, we have to select $\mu>2$.

Another benefit of the adoption of the AJM is the faster attainment of the correct scaling. It is not yet quite clear which is the true reason why the correct scaling of (14), in the region $2<\mu<3$, is significantly faster than with the SVM. One reason might be that the probability distribution of the AJM diffusion process is sharper than that of the SVM. Some more research work is required to assess this point. We hope that the results already obtained are interesting enough as to draw the attention of the researches of this field of investigation on the benefit stemming from converting the experimental data into asymmetric diffusion processes. As the last but not least remark, we want to point out that the DE method is probably the most convenient way to detect the scaling of an asymmetric diffusion processes, since this technique is not affected by the presence of biases, and does not require the adoption of any form of de-trending.

\section{References}

[1] H.E. Stanley, V. Afanasyev, L.A.N. Amaral, S.V. Buldyrev, A.L. Goldbeger, S. Havlin, H. Leschhorn, P. Maass, R.N. Mantegna, C.K. Peng, P.A. Prince, M.A. Salinger, M.H.R. Stanley, G.M. Viswanathan, Physica A 224, 302 (1996).

[2] C.-K. Peng, S.V. Buldyrev, S. Havlin, M. Simons, H.E. Stanley, and A.L. Goldberger, Phys. Rev. E, 49, 1685 (1994).

[3] C.K. Peng, S. Havlin, H.E. Stanley, A.L. Goldberger, Chaos 5, 82 (1995).

[4] N. Scafetta, P. Hamilton, P. Grigolini, in press on Fractals, condmat/0009020.

[5] P. Allegrini, M. Barbi, P. Grigolini and B.J. West, Phys. Rev. E 52, 5281 (1995). 
[6] C. Beck,F. Schlögl, Thermodynamics of Chaotic Systems, Cambridge University Press, Cambridge (1993).

[7] J.R. Dorfman, An Introduction to Chaos in Nonequilibrium Statistical Mechanics, Cambridge University Press, Cambridge (1999).

[8] P. Grigolini, N. Scafetta, V. Latora, in preparation for Phys. Rev. E

[9] M. Buiatti, P. Grigolini, L. Palatella, Physica A 268, 214 (1999).

[10] P. Allegrini, P. Grigolini, B.J. West, Phys. Rev. E 54, 4760 (1996).

[11] G. Zumofen, J. Klafter, Phys. Rev. E 47, 851 (1993).

[12] M.F. Shlesinger, J. Stat. Phys. 10, 421 (1974).

[13] E.W. Montroll, G.H. Weiss, J. Math. Phys. 6, 178 (1965).

[14] E. Barkai, R. Metzler, J. Klafter, Phys. Rev. E 61, 132 (2000).

[15] P. Lévy, Théorie de l'addition des variables aléatoires, Gauthier-Villars, Paris (1954).

[16] B.V. Gnedenko, and A.N. Kolmogorov Limit Distributions for Sums of Random Variables, Addison-Wesley, Reading, MA (1954).

[17] W. Feller, Trans. Am. Math. Soc. 67, 98 (1949).

[18] M. Bologna, P.Grigolini, M. Karagiorgis. A. Rosa, Trajctory versus probability density entropy, submitted to Phys. Rev. E

[19] M.Ignaccolo, P.Grigolini, and A. Rosa, Sporadic Randomness: The transition from the Stationary to the Non-Stationary Condition, submitted to Phys. Rev. E

[20] P. Gaspard and X.J. Wang, Proc. Natl. Acad. Sci. USA 85, 4591 (1988).

[21] Goldberger AL, Amaral LAN, Glass L, Hausdorff JM, Ivanov PCh, Mark RG, Mietus JE, Moody GB, Peng CK, Stanley HE. PhysioBank, PhysioToolkit, and Physionet: Components of a New Research Resource for Complex Physiologic Signals. Circulation 101(23): e215-e220 (Circulation Electronic Pages; http://circ.ahajournals.org/cgi/content/full/101/23/e215); 2000 (June $13)$. 


\begin{tabular}{|lc|}
\hline \multicolumn{2}{|c|}{ SVM } \\
$1<\mu<2 \quad \delta=1$ \\
$2<\mu<3 \quad \delta=1 /(\mu-1)$ \\
\hline \multicolumn{2}{|c|}{ SJM } \\
$1<\mu<2 \quad \delta=(\mu-1) / 2$ \\
$2<\mu<3 \quad \delta=1 / 2$ \\
\hline \multicolumn{2}{|c|}{ AJM } \\
$1<\mu<2 \quad \delta=\mu-1$ \\
$2<\mu<3$ & $\delta=1 /(\mu-1)$ \\
\hline
\end{tabular}

Table 1: The scaling parameter $\delta$ as a function of $\mu$ for SVM, SJM and AJM. The AJM is the only model where $\delta$ always changes upon changing $\mu$.

\begin{tabular}{|c|c|c|}
\hline Archive & $\delta$ & $\mu=\mathbf{1}+\mathbf{1} / \delta$ \\
\hline 14046 & $0.777 \pm 0.002$ & 2.29 \\
14134 & $0.863 \pm 0.006$ & 2.16 \\
14172 & $0.876 \pm 0.002$ & 2.14 \\
14184 & $0.804 \pm 0.005$ & 2.24 \\
14157 & $0.855 \pm 0.010$ & 2.17 \\
15814 & $0.872 \pm 0.002$ & 2.15 \\
\hline
\end{tabular}

Table 2: Values of the scaling parameter $\delta$ obtained with the DE method. The archive numbers correspond to the denomination of MIT-BIH LongTerm ECG Database as reported in ref.[21]. 


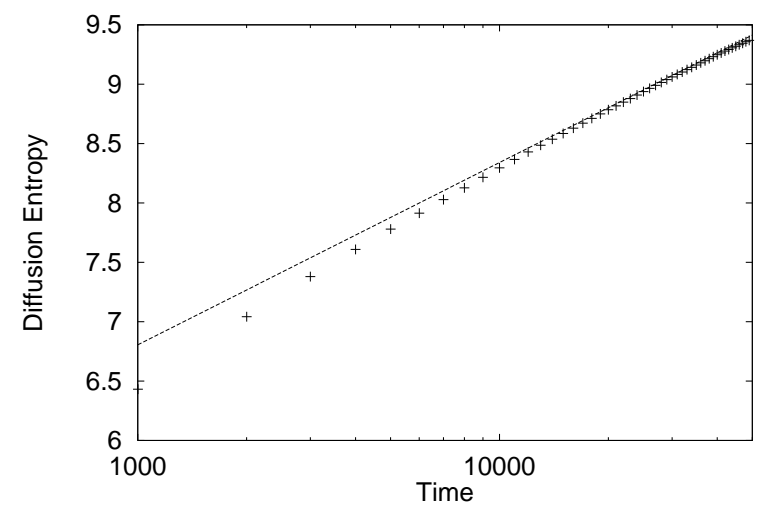

Figure 1: The diffusion entropy as a function of time. The numerical method is applied to the artificial sequence of Section 2, with $\mu=2.5$, studied according to the SVM prescription. According to the theoretical arguments of the text the scaling parameter $\delta$ is the slope of the straight line fitting the numerical results at large times, which yields in this case $\delta=2 / 3=1 /(\mu-1)$ $(T=10)$.

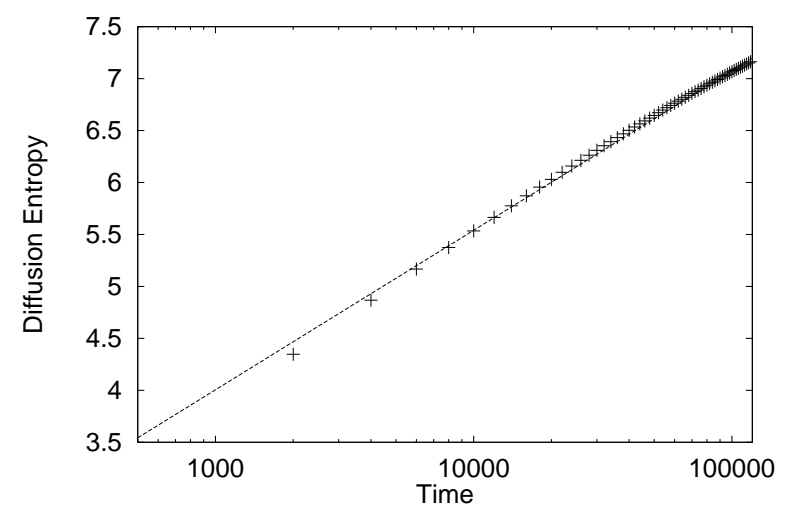

Figure 2: The diffusion entropy as a function of time. The numerical method is applied to the artificial sequence of Section 2, with $\mu=2.5$, studied according to the AJM prescription. According to the theoretical arguments of the text the scaling parameter $\delta$ is the slope of the straight line fitting the numerical results at large times, which yields in this case $\delta=2 / 3=1 /(\mu-1)$ $(T=10)$. 


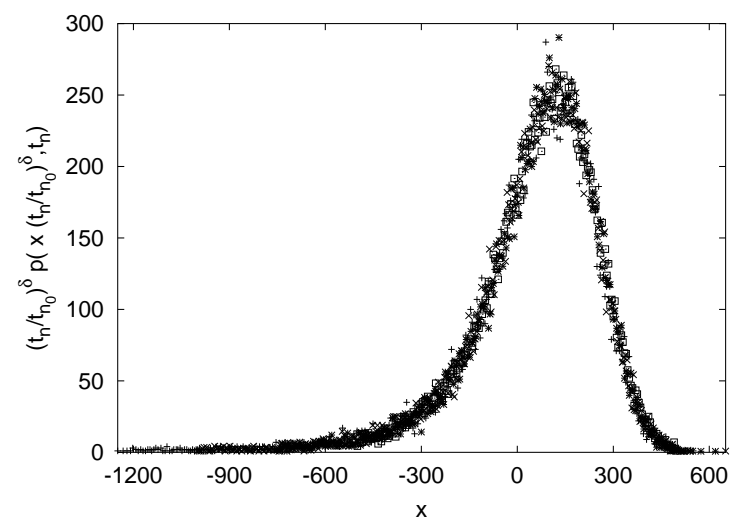

Figure 3: Scaled distributions as a function of $x$. These scaled distributions refer to the artificial sequence of Section 2 , with $\mu=2.5$, dealt with according to the AJM prescription. The times considered are: $t_{n}=2500$ '+', 2000 ' $\star$ ', 1500 ' $\times$ ', 1000 ' $\square$ '. Note that $t_{n_{0}}=1000$. Time is expressed in units of $\mathrm{T}$, with $\mathrm{T}$ defined by Eq.(13), and $\mathrm{T}=10$.

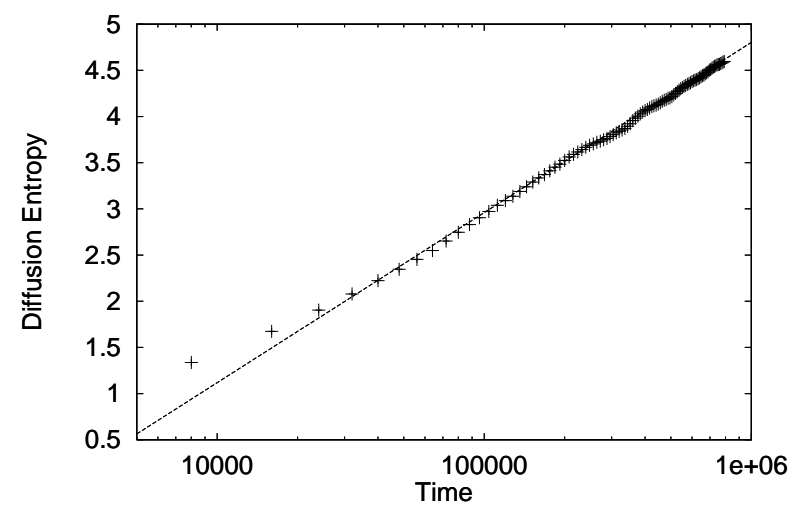

Figure 4: The diffusion entropy as a function of time. The numerical method is applied to the artificial sequence of Section 2, with $\mu=1.8$, studied according to the AJM prescription. According to the theoretical arguments of the text the scaling parameter $\delta$ is the slope of the straight line fitting the numerical results at large times, which yields in this case $\delta=\mu-1=0.8$ $(T=10)$. 


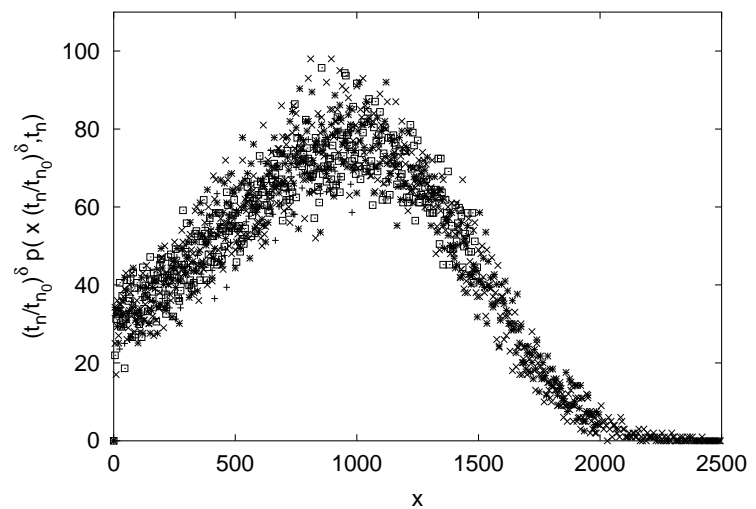

Figure 5: Scaled distributions as a function of $x$. These scaled distributions refer to the artificial sequence of Section 2 , with $\mu=1.8$, dealt with according to the AJM prescription. The times considered are: $t_{n}=2500$ '+', 2000 ' $\star$ ', 1500 ' $\times$ ', 1000 ' $\square$ '. Note that $t_{n_{0}}=1000$. Time is expressed in units of T, with $\mathrm{T}$ defined by Eq. (13), and $T=10$.

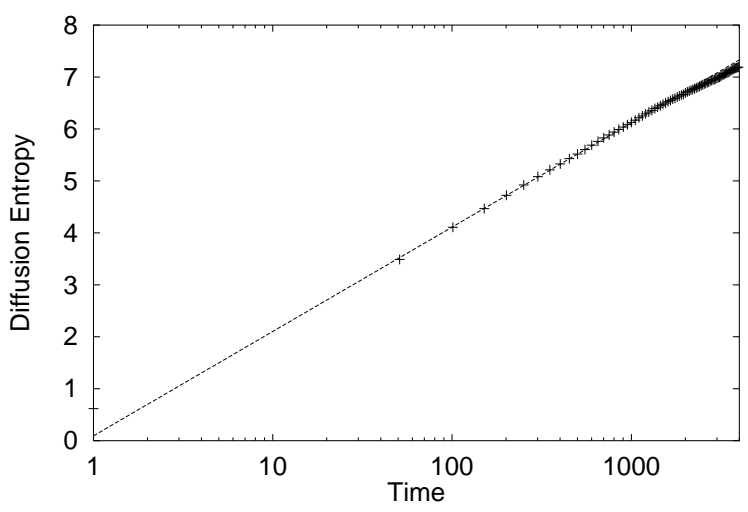

Figure 6: The DE method applied to the heart beating sequence Ltdb15814, changed into a diffusion process according to the AJM prescription. The straight line is the best fit made with Eq. (10) and it yields $\delta=0.872$. 


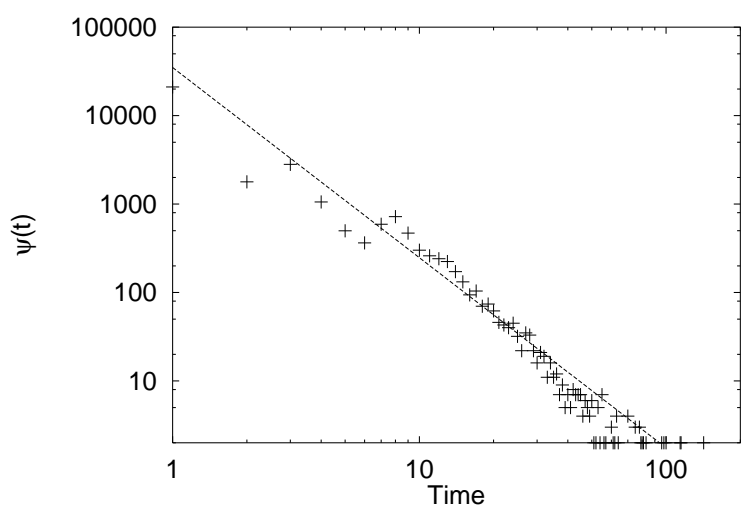

Figure 7: Coarse-grained waiting time distribution $\psi(t)$ for Ltdb15814. The straight line is an inverse powe law curve of the form $C / t^{\mu}$. According to the theory of section $3, \mu=1+1 / \delta$. 